\title{
Carboplatin-Induced Hypersensitivity Reaction
}

\author{
Evangelos Potolidis Charalampos Mandros
}

Department of Internal Medicine, Volos General Hospital, Volos, Greece

\section{Key Words}

Carboplatin · Drug hypersensitivity · Acute respiratory distress syndrome

\begin{abstract}
We describe a 65-year-old female with relapsed ovarian cancer who developed a severe hypersensitivity reaction during the second cycle of carboplatin treatment. The patient developed respiratory failure, acute respiratory distress syndrome and hypotension, requiring admission to the intensive care unit.
\end{abstract}

\section{Introduction}

Carboplatin is frequently used in patients with ovarian cancer, in second-line or salvage settings [1]. The carboplatin-associated incidence of nephrotoxicity or neurotoxicity is low. Serious hypersensitivity reactions due to carboplatin have been previously described [2-6]. Carboplatin toxicity normally develops after more than 5 or 6 cycles of treatment; however, previous studies were able to show that patients have developed toxicity during their initial therapy or during their second or third treatment [7].

\section{Case Report}

We describe a 65-year-old patient with relapsed ovarian cancer who was treated with carboplatin and developed toxicity during the second cycle. The patient received a 412-mg dose of carboplatin. The dose was calculated using the Calvert formula. Twenty min after the infusion was started, the patient developed dyspnea, wheezing, hypotension and confusion. Her blood pressure was 90/40 $\mathrm{mm}$ $\mathrm{Hg}$, saturation $78 \%$ in room air, pulse rate $140 \mathrm{bpm}$ and breath rate $34 \mathrm{breaths} / \mathrm{min}$. The infusion was stopped and our patient was transferred to the intensive care unit (ICU). She was intubated due to respiratory failure, and adrenaline, fluids and corticosteroids were infused. Cardiac ultrasound was performed and acute coronary syndrome was excluded by ECG and troponin I.

Pulmonary embolism was ruled out by a spiral CT scan of the lungs. A Swan-Ganz catheter was inserted via the right subclavian vein. Pulmonary capillary wedge pressure and central venous pressure were 4 and $3 \mathrm{~mm} \mathrm{Hg}$, respectively. The patient was ventilated based on acute respiratory 
distress syndrome ventilation protocol. Blood and urine cultures were negative, rapid abdomen ultrasound was normal and a brain CT scan was without findings. Six days after ICU admission, the patient developed fever and ventilator-associated pneumonia was diagnosed. Bronchoalveolar lavage was drawn and the patient was treated with antibiotics. Thereafter, our patient became septic and finally died.

\section{Discussion}

Carboplatin is used as a second-line drug in gynecological malignancies. Previous studies were able to document serious heterogeneous adverse hypersensitivity reactions to carboplatin administration. Toxicity due to carboplatin occurs more commonly after 5-6 cycles. Recent studies have suggested that reactions develop due to a type I response mediated by the release of histamine and cytokines as well as the involvement of a type IV reaction with T cell-mediated production of cytokines [8, 9].

Our patient developed hypotension, wheezing with respiratory failure and dyspnea during the second course of carboplatin administration, 20 min after the infusion was started. This led us to conclude that carboplatin induced a hypersensitivity reaction in our patient who received this drug due to a relapse of ovarian cancer.

Cardiogenic pulmonary edema, stroke, sepsis and pulmonary embolism were excluded. Clinicians should be alert following the initiation of carboplatin cycles. Serious adverse events normally occur after $5-6$ cycles but can develop earlier, during the first 3 treatment cycles.

\section{References}

1 Cannistra SA: Cancer of the ovary. N Engl J Med 1993;329:1550-1559.

2 Weidmann B, Mulleneisen N, Bojko P, et al: Hypersensitivity reactions to carboplatin: report of two patients, review of the literature and discussion of diagnostic procedures and management. Cancer 1994;73:2218-2222.

3 Hendrick AM, Simmons D, Cantwell BMJ: Allergic reactions to carboplatin. Ann Oncol 1992;3:239-240.

4 Morgan JS, Adams M, Mason MD: Hypersensitivity reactions to carboplatin given to patients with relapsed ovarian carcinoma. Eur J Cancer 1994;30A:1205-1206.

5 Weiss RB, Donehover RC, Wiernik PH, et al: Hypersensitivity reactions from Taxol. J Clin Oncol 1990;8:1263-1268.

-6 Sakaeda T, Kadoyama K, Yabuuchi H, Niijima S, Seki K, Shiraishi Y, Okuno Y: Platinum agent induced hypersensitivity reactions. Data mining of the FDA adverse event reporting system AERS. Int J Med Sci 2011;8:332-338.

7 Markman M, Kennedy A, Webster K, Elson P, Peterson G, Kulp B, Belinson J: Clinical features of hypersensitivity reactions to carboplatin. J Clin Oncol 1999;17:1141.

-8 Syrigou E, Syrigos K, Saif MW: Hypersensitivity reactions to oxaliplatin and other antineoplastic agents. Curr Allergy Asthma Rep 2008;8:56-62.

9 Makrilia N, Syrigou E, Kaklamanos I: Hypersensitivity reactions associated with platinum antineoplastic agents: a systematic review. Met Based Drugs 2010;2010:207084. 